\title{
Lipid Droplet Formation in Cells of the Filamentous Green Alga Klebsormidium nitens as Revealed by BODIOY-DiOC 6 and BODIPY-Nile Red Double-Staining Microscopy
}

\author{
Tsuneyoshi Kuroiwa ${ }^{1,2 *}$, Mio Ohnuma ${ }^{1,2}$, Yuuta Imoto ${ }^{1,3}$ and Haruko Kuroiwa ${ }^{1,2}$ \\ ${ }^{1}$ Faculty of Science, Rikkyo University, 3-34-1 Nishiikebukuro, Toshima-ku, \\ Tokyo 171-8501, Japan \\ ${ }^{2}$ Core Research for Evolutional Science and Technology (CREST), \\ Japan Science and Technology Agency, Gobancho, Chiyoda-ku, Tokyo 102-0076, Japan \\ ${ }^{3}$ Department of Integrated Biosciences, Graduate School of Frontier Science, \\ University of Tokyo, Tokyo 277-8562, Japan
}

Received June 26, 2014; accepted July 11, 2014

\begin{abstract}
Summary Lipid droplets (LDs) form at the surface of the endoplasmic reticulum (ER) in cells of fungi and animals. In algae, however, they appear to form at the surfaces of both the ER and plastids. To study the universality of this process, we observed LD formation in the filamentous green alga Klebsormidium nitens. Each cell of this alga contains one large petal-like chloroplast. Observations of cells stained with dipyrrometheneboron difluoride (BODIPY) revealed that small LD spots formed at the edge of the chloroplast in young cells, and the size of the spots increased as the chloroplast grew. The LD spots were distributed evenly on the chloroplast, and consequently, were distributed equally between the daughter chloroplasts after binary fission. In non-dividing starved cells, large LDs formed as the chloroplast degraded and the chlorophyll concentration decreased, suggesting that components of the chloroplast were converted into LDs as the chloroplast degraded. To examine the spatial relationships among LDs, the ER, and the chloroplast, we examined cells by microscopy after dihexyloxcacarbocyanideiodide $\left(\mathrm{DiOC}_{6}\right)$ single-staining, BODIPY-DiOC 6 double-staining, and Nile red-DiOC 6 double-staining. ERs were elongated and arranged along the edge of the chloroplast in young cells. The LDs were associated with the ER, but were absent from the area between the ER and the chloroplast. These results suggest that the ER plays an important role in LD formation in young dividing cells in the green alga. The dynamics of the ER in starved cells were obscured by the large LDs that filled the cytoplasm.
\end{abstract}

Key words Lipid droplet (LD), BODIPY-DiOC 6 double-staining, Klebsormidium nitens, Endoplasmic reticulum (ER).

The synthetic site of lipid droplets (LDs) in cells differs among various organisms. In animals and fungi, LDs are often located close to the ER, mitochondria, and peroxisomes in adipocytes and non-adipocytes (Stemberger et al. 1984, Schrader 2001, Suzuki et al. 2011). The most popular hypothesis, as summarized by Suzuki et al. (2011), is that lipid esters deposited within the ER membrane bud as a globule covered by a cytoplasmic phospholipid monolayer, but in nonadipocytes, most lipid esters are synthesized in the ER. Mitochondria and peroxisomes must have a close physical association with LDs to import fatty acids for $\beta$-oxidation. In Arabidopsis thaliana, there is a similar close relationship between LDs and glyoxysomes (peroxisomes), in which $\beta$-oxidation and the glyoxylate cycle occur (Hayashi et al. 2001). The LD-ER and LDmitochondria associations are more distant in mature adipocytes (Fawcett 1994). This may be

* Corresponding author, e-mail: tsune@rikkyo.ne.jp

DOI: $10.1508 /$ cytologia. 79.501 


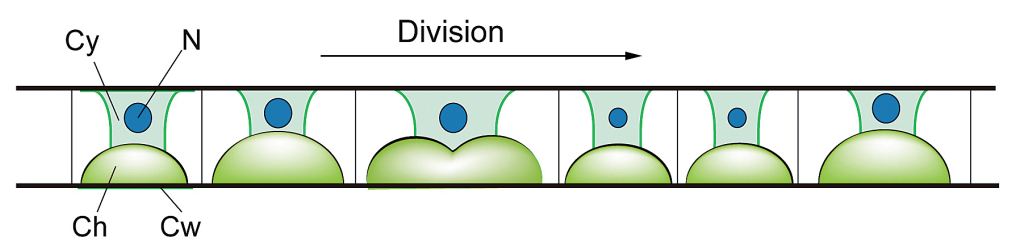

Fig. 1. Schematic illustration of chloroplast division in K. nitens. Chloroplast is often folded at one end of the cell. Chloroplast changes from oblong-like shape to football shape, then into dumbbell shape before daughter chloroplasts are pinched off. Cy, cytoplasm; N, cell nucleus; Ch, chloroplast; Cw, cell wall.

because most of the fatty acids liberated from the LD are not consumed but are supplied to other tissues (Suzuki et al. 2011).

These results and observations from previous studies suggest that the localization of LDs in cells is related to the cell condition. In cells of Chlamydomonas reinhardtii, LDs are associated with the chloroplast and the ER (Goodson et al. 2011). In our previous study, we observed that LDs were closely associated with the chloroplast periphery in nitrogen-starved C. reinhardtii cells (Kuroiwa et al. 2012). When the chloroplasts began to decompose, large LDs formed (Kuroiwa et al. 2014), indicating that in C. reinhardtii cells, chloroplast components are converted to LDs. Goodson et al. (2011) proposed that LDs in C. reinhardtii are synthesized from both the ER and chloroplasts. In the primitive red alga Cyanidioschyzon merolae, LDs are associated with only the ER (Kuroiwa et al. 2012). These results suggest that the formation of LDs is related to both chloroplast growth (in young cells) and chloroplast degradation (in starved cells).

We are interested in the universality of the formation site of LDs among eukaryotes. To explore this topic, it is important to determine the relationship between ER and LDs in green algae. However, in unicellular green algae with cup-shaped or irregularly shaped chloroplasts, it is difficult to observe the LD formation site, and to trace the behavior of LDs after formation. The cells of Klebsormidium nitens offer unique advantages for studies on LDs formation because each cell contains a single petal-like chloroplast. The chloroplast proliferates by binary fission at its central region, yielding two daughter cells. The divided cells of $K$. nitens are arranged linearly in a narrow unbranched filament enclosed in a tubular cell wall; therefore, daughter cells and organelles can be traced clearly under a microscope (Fig. 1). Also, green algae with large nuclear genomes tend to form large LDs (Kuroiwa et al. 2012). Indeed, K. nitens and Klebsormidium flaccidum have large nuclear genomes of $310.8 \mathrm{Mbp}$ (Kuroiwa et al. 2012) and 117.1 Mbp (Hori et al. 2014), respectively, and $K$. nitens cells form large LDs. Thus, it is easy to observe LD formation in $K$. nitens (Kuroiwa et al. 2012).

In this study, we treated $K$. nitens cells using dipyrrometheneboron difluoride (BODIPY)dihexyloxcacarbocyanideiodide $\left(\mathrm{DiOC}_{6}\right)$ and BODIPY-Nile red double-staining methods. Microscopic analyses of the stained cells showed that LDs form at the edge of the ER, but never at sites between the ER and the chloroplast periphery. The results suggest that in this green alga, LDs form at the ER, like in red algae (Kuroiwa et al. 2012).

Materials and methods

\section{Culture}

$K$. nitens cells were collected from the surface of an aquarium and cultured in fresh water at $23^{\circ} \mathrm{C}$ under continuous light $\left(40 \mu \mathrm{mol}\right.$ photons $\mathrm{m}^{-2} \mathrm{~s}^{-1}$ ) (Kuroiwa et al. 2012). The algal cells were processed for microscopic observations after 2 and $10 \mathrm{~d}$ of culture. 

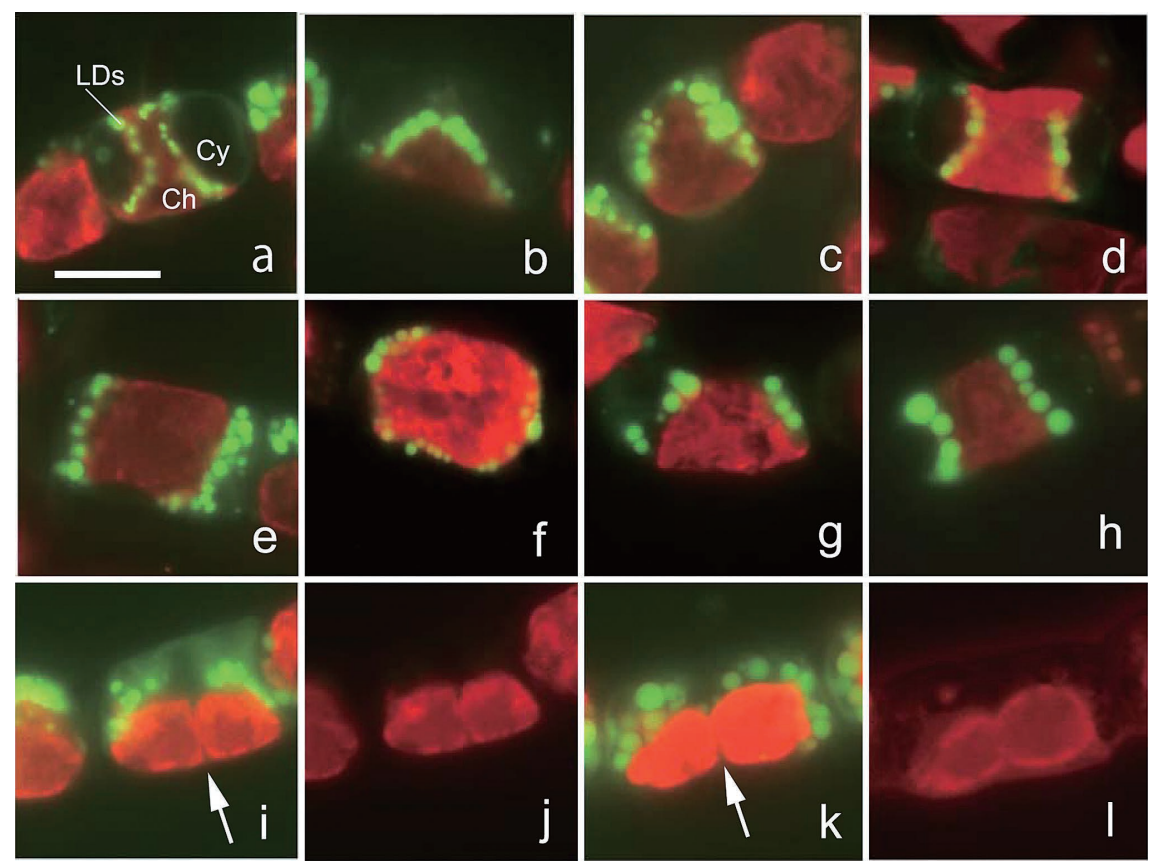

Fig. 2. Localization of lipid droplets at chloroplast periphery at various stages as observed by BODIPY staining. a-h: Fluorescence images of chloroplasts and LDs. Size of LDs increased as chloroplasts grew. i, j and k, l: Fluorescence images of dividing chloroplasts and omit the same fields. i, k show violet-light excitation, j, 1 show red-light excitation. LDs, lipid droplets; Cy, cytoplasm; Ch, chloroplast. Arrows show chloroplast-division sites. Scale bars: $10 \mu \mathrm{m}$.

\section{Fluorescence microscopy}

To observe LDs, cells were stained using dipyrrometheneboron difluoride (BODIPY). The cells were stained for $5 \mathrm{~min}$ with $1 \mu \mathrm{g} / \mathrm{mL}$ BODIPY in TAN buffer $(20 \mathrm{mM}$ Tris- $\mathrm{HCl}, 0.5 \mathrm{mM}$ EDTA, $1.2 \mathrm{mM}$ spermidine) as described previously (Kuroiwa et al. 2012). BODIPY was first dissolved in ethanol $(1 \mathrm{mg} / \mathrm{mL})$ and then diluted in TAN buffer. A $5-\mu \mathrm{L}$ aliquot of the BODIPY mixture was placed onto a clean slide glass and then a $3-\mu \mathrm{L}$ cell sample was added. The mixture was mixed gently, and then covered with a coverslip. Fresh BODIPY solution was added to the samples from the edge of the coverslip. The edges of the coverslip were sealed with nail polish. Chloroplasts emitted red autofluorescence. The ER emitted yellow-green fluorescence $(550 \mathrm{~nm})$

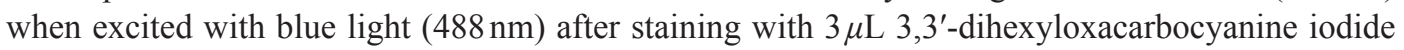
$\mathrm{DiOC}_{6}$ (a dye specific for cellular membranes), as described elsewhere (Terasaki et al. 1984, Kawazu et al. 1995).

For BODIPY-DiOC 6 double-staining, $3 \mu \mathrm{L}$ DiOC $_{6}$ stock solution was mixed with $3 \mu \mathrm{L}$ BODIPY in TAN buffer, and the resulting $6-\mu \mathrm{L}$ mixture was used as the staining solution. LDs were visualized as yellow-green fluorescence when excited with violet light $(430 \mathrm{~nm})$.

For $\mathrm{DiOC}_{6}$-Nile red double-staining, Nile red was first dissolved in acetone $(1 \mathrm{mg} / \mathrm{mL})$ and then diluted with phosphate buffer $(20 \mu \mathrm{g} / \mathrm{mL})$ (Fowler and Greenspan 1985). Then, a 3- $\mu \mathrm{L}$ aliquot of the Nile red stock solution was mixed with $3 \mu \mathrm{L} \mathrm{DIOC}_{6}$ in TAN buffer and the mixture was used as the staining solution. The ER and LDs were visualized as yellow-green fluorescence and yellow red fluorescence, respectively, when excited with violet light.

Cells were viewed under an epifluorescent microscope (BX51; Olympus, Tokyo, Japan) and images were acquired with a 3CCD digital camera (C7780; Hamamatsu Photonics, Hamamatsu, Japan) under violet (for ER and LDs) or green excitation $(568 \mathrm{~nm}$ ) (for chloroplast autofluorescence). 

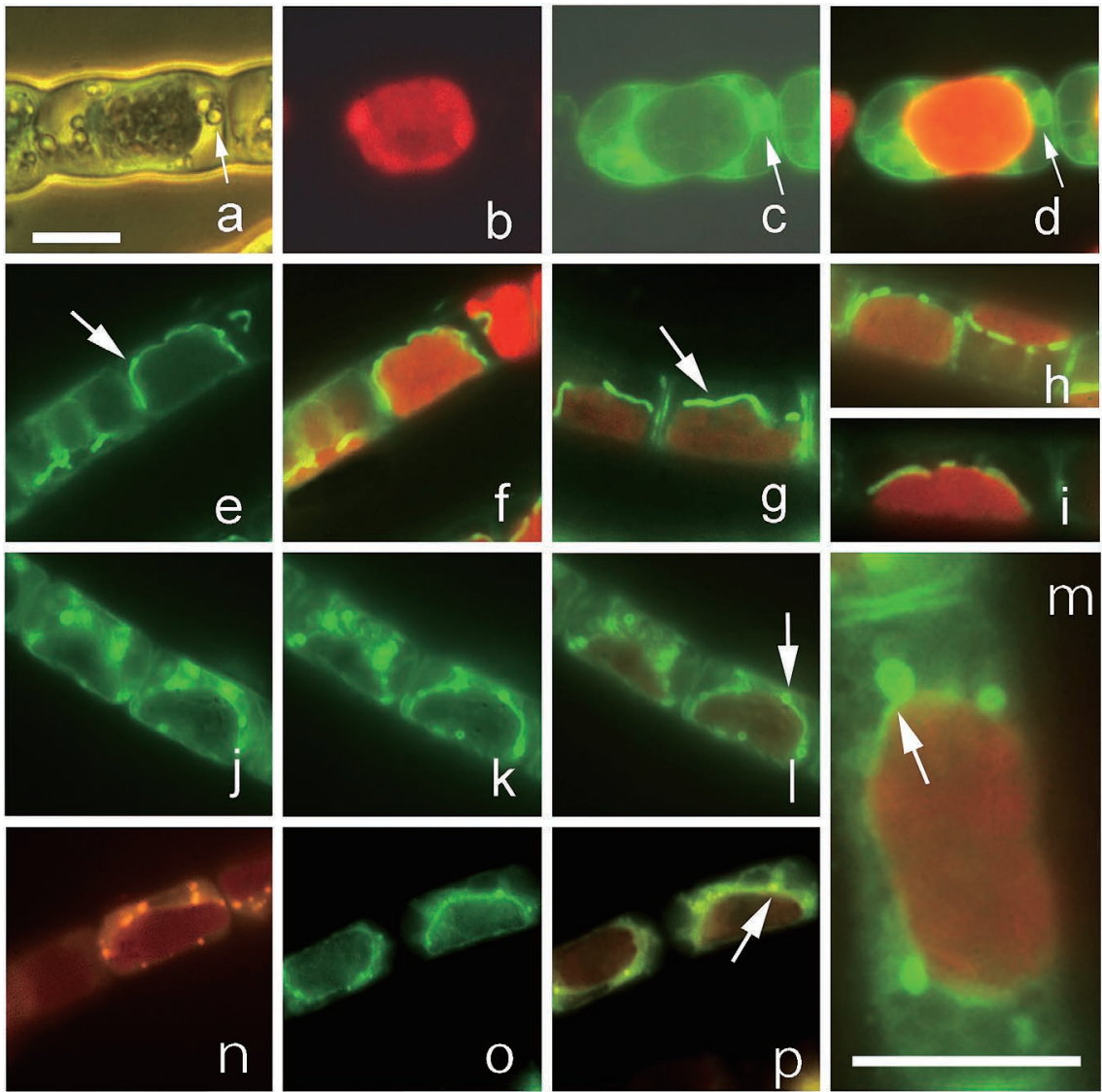

Fig. 3. Membrane localization at chloroplasts and lipid droplets observed after staining with $\mathrm{DiOC}_{6}$. a-d show different images of the same field as follows; phase-contrast image (a) and fluorescence images after green- (b), blue- (c) and violet-light excitation (d). LD surface (arrow) was stained weaker than cytoplasmic membrane systems including chloroplast envelope. e-i: Fluorescence images of endoplasmic reticulum (arrows) and $\mathrm{LDs}$ after $\mathrm{DiOC}_{6}$ staining, as follows: e, f: fluorescence images of the same field after excitation with blue light (e) and violet light (f). ERs appear to associate with edge of chloroplasts (arrow). Similar results were observed in cells excited by violet light (arrows in $\mathrm{g}$, h, and i). $\mathrm{j}-\mathrm{m}$ : Fluorescence images of ERs, LDs, and chloroplasts after double-staining with $\mathrm{DiOC}_{6}$ and BODIPY. $\mathrm{j}-1$. Same field excited with blue (j, k) and violet lights (1). Focus for $\mathrm{j}$ differed slightly from focus for $\mathrm{k}$ and $\mathrm{l}$. LDs were located on ER (arrow). Location of LDs on ER was also shown in a higher magnification image (arrow in $\mathrm{m}$ ). $\mathrm{n}$ : Fluorescence image of chloroplast and LDs (red-yellow) after staining with Nile Red. o, p. Fluorescence images of chloroplast and LDs (yellow). Figures show the same field after double-staining with BODIPY and Nile Red. LD was associated with ER (arrow). The chloroplast emitted red autofluorescence. Scale bars: $10 \mu \mathrm{m}$.

\section{Results and discussion}

\section{Formation of LDs at chloroplast periphery in young dividing cells}

The cells of $K$. nitens were parallelepiped (Fig. 1). Each cell contained a roughly oblong chloroplast bent along one side of the cell. The chloroplast formed into a football shape, then a dumbbell shape, and then divided into daughter chloroplasts. After young K. nitens cells were stained with BODIPY, we observed many small spherical LDs of a similar size $(0.7 \mu \mathrm{m}$ in diameter) closely arranged around the chloroplast periphery (Fig. 2a). The chlorophyll fluorescence 

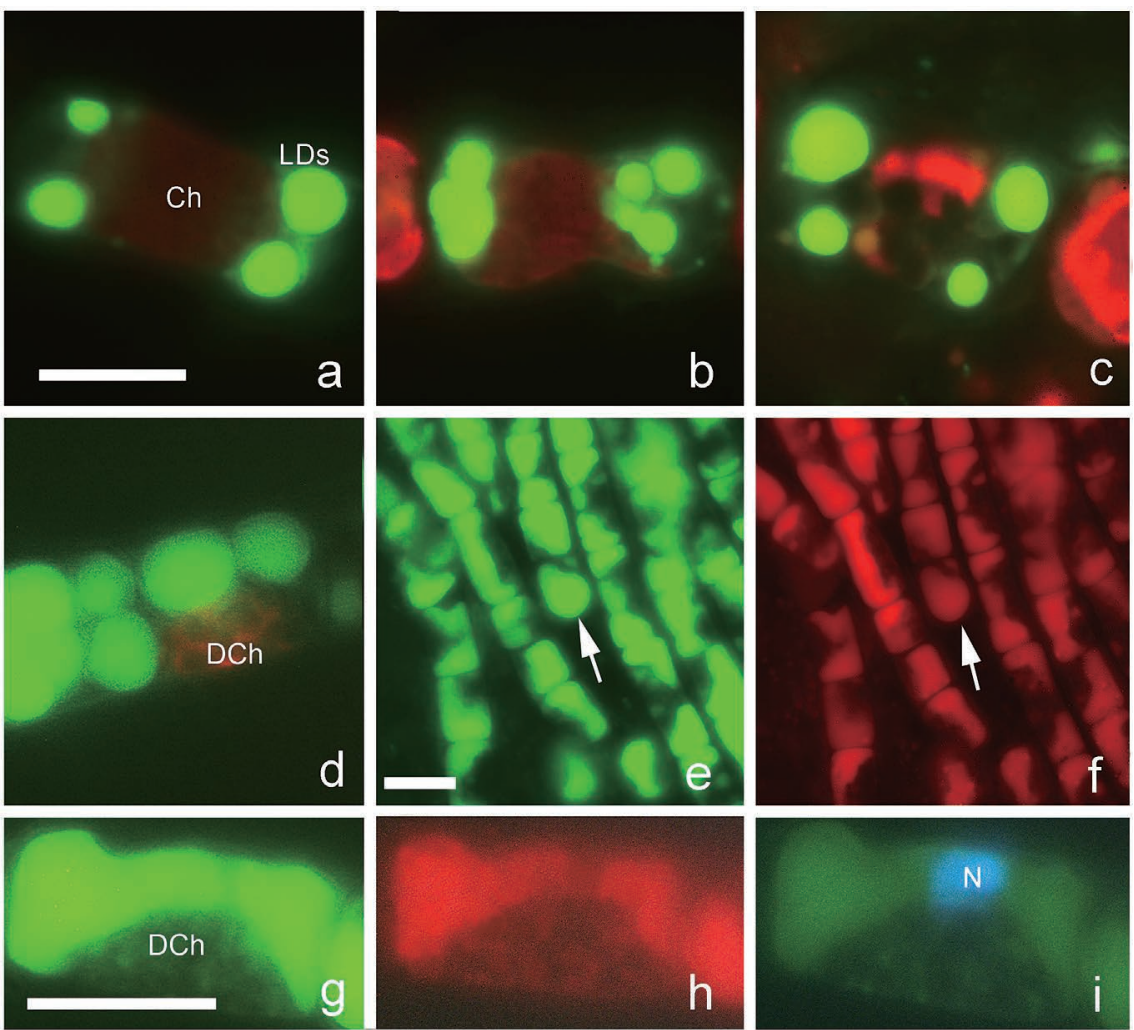

Fig. 4. Appearance of large LDs in starved cells. a-i: Fluorescence images of chloroplast and lipid droplets in cells after staining with BODIPY $(\mathrm{a}-\mathrm{f})$ and double-staining with BODIPY and DAPI (g-i). Large LDs appeared as amount of chlorophyll in chloroplasts decreased (a-d). Some large LDs were not associated with chloroplasts. e, f. Same field at low magnification; chlorophylls moved from chloroplasts to cytoplasm (arrow) where LDs were present. g-i. Same field excited by violet (g), red (h) and ultraviolet light (i). Cell nucleus remained a spherule. Ch, chloroplast; LDs, lipid droplets; DCh, disintegrated chloroplast; N, cell nucleus. Scale bars: $10 \mu \mathrm{m}$.

was dense. Each LD grew as the size of the chloroplast increased (Fig. $2 b-h$ ). LDs were present at the peripheries of the daughter chloroplasts (Fig. 2i-1). There was a similar number of LDs, all with a similar size, on each daughter chloroplast. There were no large LDs on the daughter chloroplasts, suggesting that the LDs were not synthesized actively on the dividing chloroplast.

\section{Formation of LDs on ER associated with chloroplasts}

The cells were stained with $\mathrm{DiOC}_{6}$ and excited by violet light to observe the chloroplast and LDs by phase-contrast microscopy (Fig. 3a). The chloroplast emitted red autofluorescence (Fig. $3 \mathrm{~b}, \mathrm{~d})$. The chloroplast envelopes emitted green fluorescence, and the surface of LDs appeared to emit weak fluorescence (Fig. 3c,d). Staining of cells with the membrane-staining dye DiOC $_{6}$ revealed string-like ERs (Fig. 3e), most of which were arranged around the edge of the chloroplasts (Fig. $3 \mathrm{f}-\mathrm{i}$ ). Double-staining of LDs and ERs with BODIPY and $\mathrm{DiOC}_{6}$ revealed that LDs were located at the edge of the cytoplasmic side of the ERs, but not between ERs and chloroplasts (Fig. $3 j-m)$. When the cells were stained with Nile red, the LDs associated with the chloroplast edges emitted yellow-red fluorescence (Fig. 3n). We confirmed this observation by double-staining cells with $\mathrm{DiOC}_{6}$ and Nile Red. The stained ERs and LDs emitted green and yellow-red fluorescence, respectively. These results confirmed that the LDs were located on the outside of the ERs and the 

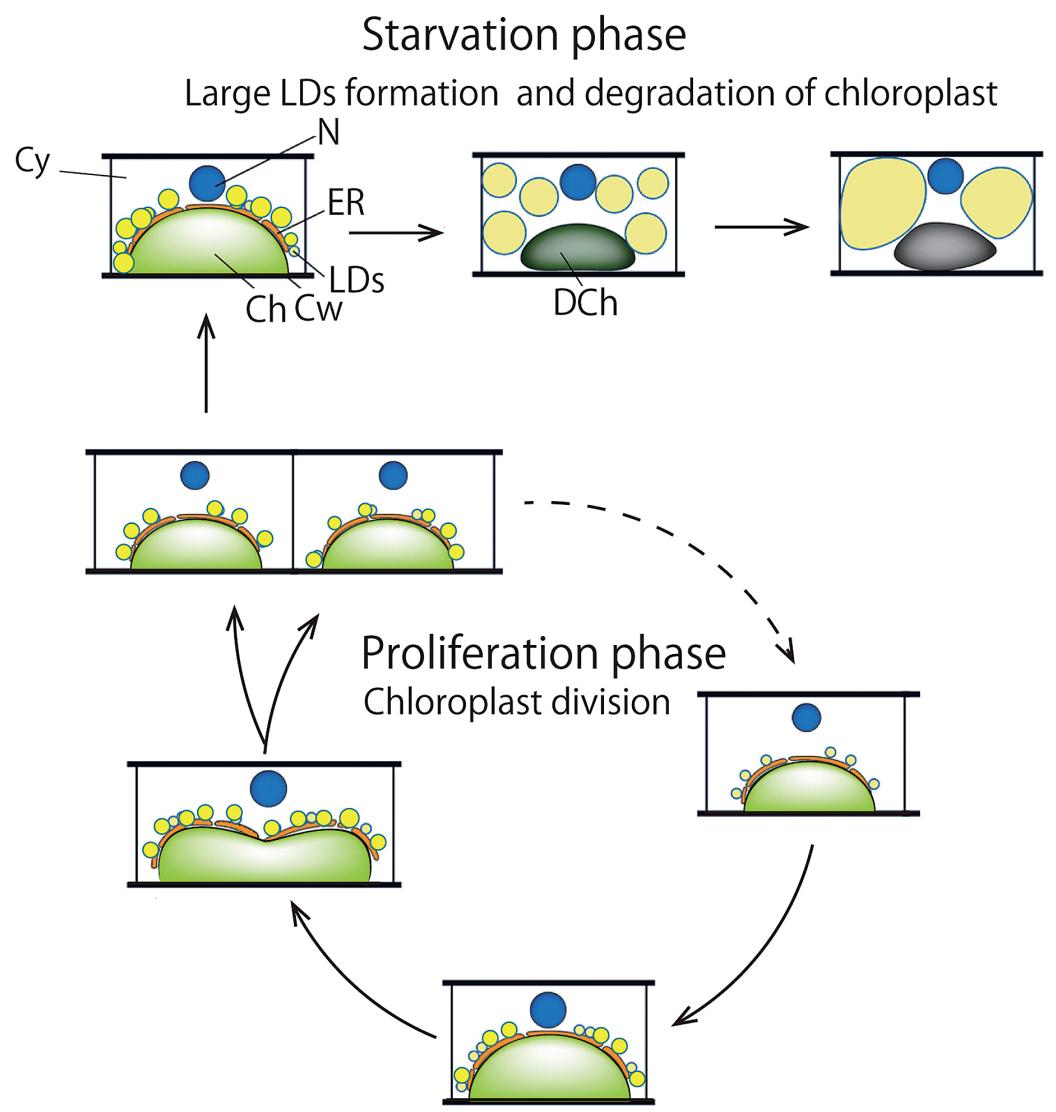

Fig. 5. Schematic depiction of lipid droplets formation in K. nitens. In dividing cells, LDs formed at the endoplasmic reticulum located at chloroplast edges. In starved cells, chloroplasts began to disintegrate and large LDs formed not only at the ER but also in cytoplasm detached from ER. In some cells, LDs fused to form large LDs. Chlorophylls move from chloroplast to cytoplasm as chloroplast degrades, but nucleus remains a spherule. $\mathrm{Cy}$, cytoplasm; N, cell nucleus; ER, endoplasmic reticulum; LDs, lipid droplets; Ch, chloroplast; $\mathrm{Cw}$, cell wall; $\mathrm{DCh}$, disintegrated chloroplast.

chloroplast (Fig. 3n-p). These findings also showed that LDs formed on the ER in these green alga cells, as is the case in red alga (Kuroiwa et al. 2012), fungi (Murphy and Vance 1999), and animals (Suzuki et al. 2011). The hypothesis that LDs form from a unit membrane of the ER, which was proposed for animal cells (Suzuki et al. 2011), also appears to hold true for algae. Therefore, this may be the general mechanism of LD formation.

\section{Formation of large LDs in starved cells}

In our previous study, we showed that formation of large LDs in C. reinhardtii was accompanied by chloroplast decomposition and chlorophyll loss (Kuroiwa and Kuroiwa 2014), suggesting that chloroplast components are converted into LDs. In $K$. nitens cells, large LDs appeared in cells cultured for $10 \mathrm{~d}$. Some of the large LDs were not associated with chloroplasts, and the chlorophyll fluorescence in the disintegrated chloroplast was weak or absent (Fig. 4a-d). Spherical LDs fused to form large LDs that filled the cytoplasm (Fig. 4e, g). The chlorophyll fluorescence was superimposed on that of LDs, although the cell nucleus remained as a spherule (Fig. 4e-i). These results support the idea that materials from degrading chloroplasts may be involved in LD formation (Wanner et al. 1981, Kuroiwa et al. 2014). 
Our data indicate that LDs form at two stages during the life of the cell: during the formation of chloroplasts in young dividing cells, and during the degradation of chloroplasts in starved cells (Fig. 5). First, small LDs form at the ERs, and are distributed into the daughter cells because they remain attached to the ER surrounding the dividing chloroplast in young cells. Second, the components of degraded chloroplasts are converted into LDs, which fuse to form larger LDs in starved cells (Fig. 5). These hypotheses should be tested in further studies using electron microscope analyses.

\section{Acknowledgments}

This work was supported by a Grant-in-Aid for Scientific Research (A) (2224707).

\section{References}

Fawcett, D. W. 1994. A Textbook of Histology. Chapman \& Hall, New York.

Fowler, S. D. and Greenspan, P. 1985. Application of Nile red, a fluorescent hydrophobic probe, for the detection of neutral lipid deposits in tissue sections: Comparison with oil red O. J. Histochem. Cytochem. 33: 833-836.

Goodson, C., Roth, R., Wang Z. T. and Goodenough U. 2011. Structural correlates of cytoplasmic and chloroplast lipid body synthesis in Chlamydomonas reinhardtii and stimulation of lipid body production with acetate boost. Eukaryotic Cell 10: 1592-1606.

Hayashi, Y., Hayashi, M., Hayashi, H., Hara-Nishimura, I. and Nishimura, M. 2001. Direct interaction between glyoxysomes and lipid bodies in cotyledons of the Arabidopsis thaliana ped1 mutant. Protoplasma 218: 83-94.

Hori, K., Maruyama, F., Fujisawa, T., Togashi, T., Yamamoto, N., Seo, M., Sato, S., Yamada, T., Mori, H., Tajima, N., Moriyama, T., Ikeuchi, M., Watanabe, M., Wada, H., Kobayashi, K., Saito, M., Masuda, T., Sekimoto, Y. S., Mashiguchi, K., Awai, K., Shimojima, M., Masuda, S., Iwai, M., Nobusawa, T., Narise, T., Kondo, S., Saito, H., Sato, R., Murakawa, M., Ihara, Y., Yamada, Y. O., Ohtaka, K., Satoh, M., Sonobe, K., Ishii, M., Ohtani, R., Sato, M. K., Honoki, R., Miyazaki, D., Mochizuki, H., Umetsu, J., Higashi, K., Shibata, D., Kamiya, Y., Sato, N., Nakamura, Y., Tabata, S., Ida, S., Kurokawa, K. and Ohta, H. 2014. Klebsormidium flaccidum genome reveals primary factors for plant terrestrial adaptation. Nat. Commun. 16: 1-9.

Kawazu, T., Kawano, S. and Kuroiwa, T. 1995. Distribution of the Golgi apparatus in the mitosis of cultured tobacco cells as revealed by $\mathrm{DiOC}_{6}$ fluorescence microscopy. Protoplasma 186: 183-192.

Kuroiwa, T., Ohnuma, M., Imoto, Y., Misumi, O., Fujiwara, T., Miyagishima, S. Y., Sumiya, N. and Kuroiwa, H. 2012. Lipid droplets of bacteria, algae and fungi and a relationship between their contents and genome sizes as revealed by BODIPY and DAPI Staining. Cytologia 77: 289-299.

Kuroiwa, H. and Kuroiwa, T. 2014. Production of oil bodies in response to nitrogen starvation in Chlamydomonas reinhardtii. In: Noguchi, T., Kawano, S., Tsukaya, H., Matsunaga, S., Sakai, A., Karahara, I. and Hayashi, Y. (eds.). Atlas of Plant Cell Structure. Springer, Tokyo. p. 104.

Murphy, D. J. and Vance, J. 1999. Mechanisms of lipid-body formation. Trends Biochem. Sci. 24: 109-115.

Schrader, M. 2001. Tubulo-reticular clusters of peroxisomes in living COS-7 cells: Dynamic behavior and association with lipid droplets. J. Histochem. Cytochem. 49: 1421-1429.

Stemberger, B. H., Walsh, R. M. and Patton, S. 1984. Morphometric evaluation of lipid droplet associations with secretory vesicles, mitochondria and other components in the lactating cell. Cell Tissue Res. 236: 471-475.

Suzuki, M., Shinohara, Y., Ohsaki, Y. and Fujimoto, T. 2011. Lipid droplets: size matters. J. Electron Microsc. (Tokyo) 60: S101-S116.

Terasaki, M., Song, J. D., Wong, J. R., Weiss, M. J. and Chen, L. B. 1984. Localization of endoplasmic reticulum in living and glutaraldehyde-fixed cells with fluorescent dyes. Cell 38: 101-108.

Wanner, G., Formanek, H. and Theimer, R. R. 1981. The ontogeny of lipid bodies (spherosomes) in plant cells. Planta 159: $109-123$. 